\title{
Valeur Ethnobotanique De Annona Muricata Utilisee Dans Le Traitement De Certaines Pathologies Au Sud-Benin
}

\author{
Arnaud N. Kohonou, Msc \\ Nicodème Worou Chabi, PhD
}

Laboratoire de Recherche en Biologie Appliquée, École Polytechnique d'Abomey-Calavi, Université d'Abomey-Calavi ,Cotonou, Bénin

\section{Durand Dah-Nouvlessounon, PhD \\ Sina Haziz, PhD}

Laboratoire de Biologie et de Typage Moléculaire en Microbiologie,

Département de Biochimie et Biologie Cellulaire, FAST/UAC, Cotonou, Bénin

\section{Victorien Dougnon, PhD}

Unité de Recherche en Microbiologie Appliquée et Pharmacologie des substances naturelles (URMAPHa), Laboratoire de Recherche en Biologie Appliquée (LARBA), Ecole Polytechnique d'Abomey-Calavi (EPAC),

Université d'Abomey-Calavi, Cotonou, Bénin

\section{Martial Nounagnon, PhD}

Laboratoire de Biologie et de Typage Moléculaire en Microbiologie,

Département de Biochimie et Biologie Cellulaire, FAST/UAC, Cotonou, Bénin

Basile G. Sognigbé, Msc

Laboratoire de Recherche en Biologie Appliquée, École Polytechnique d'Abomey-Calavi, Université d'Abomey-Calavi, Cotonou, Bénin

\section{Pacôme A. Noumavo, PhD \\ Lamine Baba-Moussa, PhD}

Laboratoire de Biologie et de Typage Moléculaire en Microbiologie,

Département de Biochimie et Biologie Cellulaire, FAST/UAC,

Cotonou, Bénin

\section{Résumé}

L'utilisation d'Annona muricata dans le traitement de diverses pathologies humaines prend de plus en plus d'ampleur au Bénin. Le but de la présente étude est de faire l'inventaire des maladies traitées par l'utilisation de cette plante. Pour ce faire, une enquête ethnobotanique a été réalisée auprès de 
77 herboristes et 170 tradithérapeutes des Départements de l'Ouémé et du Plateau, du Littoral, de l'Atlantique et du Mono. Les données collectées étaient relatives à la structure écologique de l'arbre Annona muricata, aux différents organes utilisés et les maladies traitées, au mode d'emploi, etc. Ces données ont été analysées avec le logiciel SPSS (Statistical Package for Social Sciences) pour la détermination des statistiques descriptives en termes de pourcentage et de moyenne. Il ressort de l'étude que les acteurs enquêtés étaient majoritairement des hommes (74,9\%),non scolarisés (40,1\%), d'origine Goun (27,1\%), Cotafon $(19,8 \%)$ et Nago $(18,2 \%)$. Les caractéristiques écologiques de $A$. muricata ont varié significativement $(\mathrm{p}<0,05)$ selon les enquêtés. Le mode de multiplication de l'arbre, selon les enquêtés,se fait principalement par la graine $(82,6 \%)$. Les principaux organes d'Annonamuricata utilisés pour le traitement des maladies sont majoritairement le fruit $(86,2 \%)$, la feuille $(66,4 \%)$ et l'écorce $(62,8 \%)$. Les principaux modes de préparation des organes étaient la tisane $(97,2 \%)$, ladécoction $(93,5 \%)$, et la poudre $(76,9 \%)$. L'Annonamuricata était majoritairement utilisé, selon les enquêtés, pour le traitement de l'insomnie $(70,9 \%)$, le cancer $(70 \%)$, le paludisme $(59,9 \%)$, l'hypertension artérielle $(58,7 \%)$, le vertige $(57,5)$...Ainsi, vu les résultats, ce travail a permis de connaitre la valeur ethnobotanique d'Annona muricata.

Mots clés : Annonamuricata, Tradithérapeutes, Maladies, Cancer, Benin 


\title{
Ethnobotanical Value of Annona Muricata Used in the Treatment of Certain Pathologies in South Benin
}

\author{
Arnaud N. Kohonou, Msc \\ Nicodème Worou Chabi, PhD
}

Laboratoire de Recherche en Biologie Appliquée, École Polytechnique

d'Abomey-Calavi, Université d'Abomey-Calavi ,Cotonou, Bénin

Durand Dah-Nouvlessounon, PhD

Sina Haziz, PhD

Laboratoire de Biologie et de Typage Moléculaire en Microbiologie,

Département de Biochimie et Biologie Cellulaire, FAST/UAC,

Cotonou, Bénin

Victorien Dougnon, PhD

Unité de Recherche en Microbiologie Appliquée et Pharmacologie des substances naturelles (URMAPHa), Laboratoire de Recherche en Biologie

Appliquée (LARBA), Ecole Polytechnique d'Abomey-Calavi (EPAC),

Université d'Abomey-Calavi, Cotonou, Bénin

\section{Martial Nounagnon, PhD}

Laboratoire de Biologie et de Typage Moléculaire en Microbiologie,

Département de Biochimie et Biologie Cellulaire, FAST/UAC,

Cotonou, Bénin

Basile G. Sognigbé, Msc

Laboratoire de Recherche en Biologie Appliquée, École Polytechnique

d'Abomey-Calavi, Université d'Abomey-Calavi, Cotonou, Bénin

\section{Pacôme A. Noumavo, PhD \\ Lamine Baba-Moussa, PhD}

Laboratoire de Biologie et de Typage Moléculaire en Microbiologie,

Département de Biochimie et Biologie Cellulaire, FAST/UAC, Cotonou, Bénin

\begin{abstract}
The use of Annona muricata(A. muricata) in the treatment of various human pathologies is becoming more and more widespread in Benin. The aim of this study was to inventory the diseases treated by the use of this plant. For this purpose, an ethnobotanical survey was carried out among 77 herbalists and 170 traditional therapists from the Departments of Ouémé and Plateau, Littoral, Atlantic and Mono. The data collected was relative, to the ecological
\end{abstract}


structure of the Annona muricata tree, the different organs used and the diseases treated, the use instructions, etc. The data was collected in the form of a questionnaire. These data had been analysed with the Statistical Package for Social Sciences (SPSS) software for the determination of descriptive statistics in terms of percentage and average. The study showed that the majority of the actors surveyed were men (74.9\%), unschooled (40.1\%), ethnic groups as Goun $(27.1 \%)$, Cotafon $(19.8 \%)$ and Nago (18.2\%). The ecological characteristics of $A$. muricatavaried significantly $(\mathrm{p}<0.05)$ according to the surveyed actors. The main method of multiplication of the tree is by seeds $(82.6 \%)$. The main organs of Annona muricata used for diseases treatment were usually the fruit $(86.2 \%)$, the leaf $(66.4 \%)$ and the bark $(62.8 \%)$. According to the actors, Annona muricata was mainly used for the treatment of insomnia (70.9\%), cancer $(70 \%)$, malaria (59.9\%), high blood pressure (58.7\%), vertigo (57.5), etc. The main method of organ preparation was herbal tea $(97.2 \%)$, decoction $(93.5 \%)$, and powder $(76.9 \%)$. Therefore, at the results showed, this study allowed to know ethnobotanic value of Annona muricata.

Keywords: Annona Muricata, Traditional Therapists, Diseases, Cancer, Benin

\section{Introduction}

Le cancer est une tumeur maligne causée par une multiplication anarchique de cellules. C'est une maladie caractérisée par la prolifération incontrôlée de cellules liée à un échappement au mécanisme de régulation qui assure le développement harmonieux de notre organisme (Globocan, 2012). On estime à 14,1 millions le nombre de nouveaux cas de cancer et 8,2 millions le nombre de décès liés aux cancers survenus dans le monde en 2012(OMS, 2012).Longtemps considérée comme une « maladie de riche », le cancer frappe officiellement près de 850.000 personnes par an sur le continent africain(Aliam UICC, 2017). Au Bénin, la fréquence globale des cancers urologiques était de 17,38\%.Il s'agissait de cancers prostatiques (69\%), de vessie $(28,5 \%)$ du rein $(8,5 \%)$ et les cancers des organes génitaux externes (Egué, 2018).Dans nos hôpitaux au Bénin le nombre de malades de cancer augmente de jour en jour, la ville de Cotonou enregistre à elle seule 1500 cas de cancers chaque année dont 55\% de décès depuis 2013 (Egué, 2018). De même les agents de santé sont confrontés à des problèmes de prise en charge des malades cancéreux qui deviennent de plus en plus nombreux (Houngbé, 2018).Juste après un petit soulagement dans les hôpitaux, les patients cherchent à vite rentrer chez eux pour continuer le traitement des maladies avec la médecine traditionnelle (dont les remèdes sont à base de plantes) et même dans les cas des maladies graves comme le diabète et le cancer (Houngbé, 2018).).Environ 80\% de la population Béninoise continuent de se 
soigner en recourant à la médecine traditionnelle, (WHO,2009). D’ailleurs le Bénin est un pays qui dispose d'un héritage ethnobotanique non négligeable.

La mission de Adjanohoun et al. (1998) a recensé près de 501 espèces utilisées en médecine traditionnelle. C'est cet atout traditionnel qui fait que, par manque de moyens, la majorité des malades du cancer au Benin préfère faire recours à la médecine traditionnelle en se rendant chez les tradithérapeutes ou chez les herboristes dans les marchés, où leurs sont indiqués ou vendus les organes des plantes, utilisés en combinaison ou seuls en occurrence ceux de la plante d'Annona muricata (feuille, écorce).Ces organes sont aussi vendus à d'autres patients ne souffrant pas du cancer. Ce constat non moins négligeable nous a poussé à chercher à mieux connaitre Annona muricata, son utilité, ses vertus. A cet effet, il nous est apparu impérieux de mener une enquête ethnobotanique afin de nous convaincre sur le choix des malades qui préfèrent la médecine traditionnelle pour leurs traitements. Explorer la flore béninoise utilisée par les acteurs de la médecine traditionnelle en vue de découvrir de nouvelles molécules est désormais une alternative.

Il existe dans la littérature scientifique béninoise de nombreuses enquêtes ethnobotaniques consacrées aux plantes médicinales utilisées dans le traitement de diverses affections (Adomou et al., 2011, Agankpé et al.,2014) mais aucune ,à notre connaissance, ne s'est intéressée aux plantes vendues par les herboristes pour le traitement des cancers au Sud-Bénin .

\section{Matériel et méthodes}

\section{Cadre d'étude}

L'enquête a été réalisée au Sud-Bénin dans les Départements de l'Ouémé et du Plateau, du Littoral, de l'Atlantique et du Mono (figure 1).

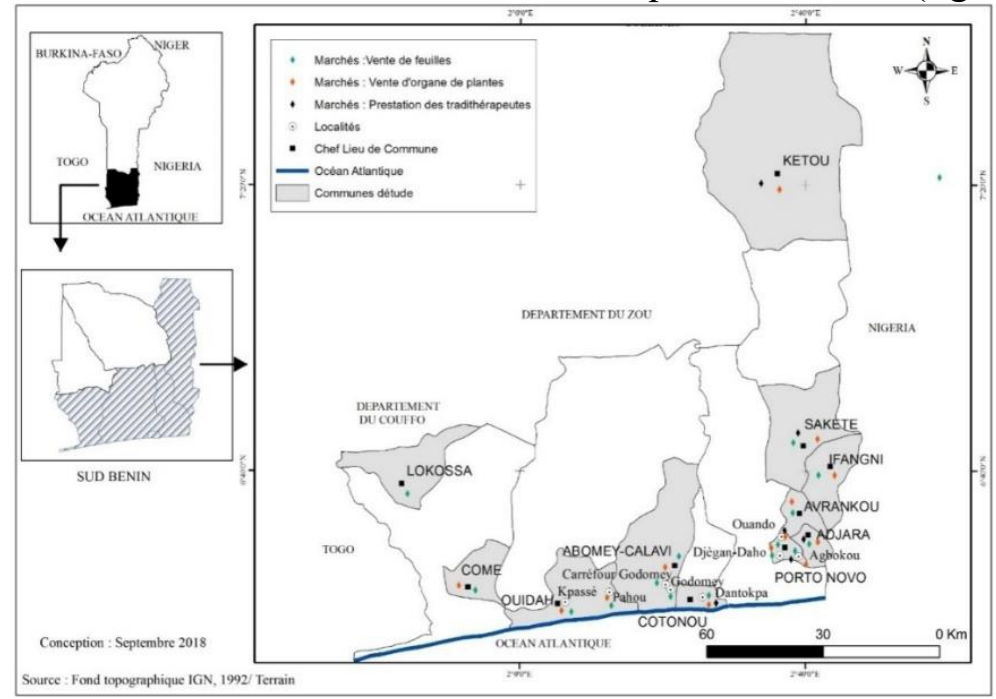

Fig. 1 : Carte de la zone d'étude 
La zone d'étude appartient à la zone Guinéo-Congolaise située entre $6^{\circ} 25^{\prime} \mathrm{N}$ et $7^{\circ} 30^{\prime} \mathrm{Net}$ caractérisée par quatre saisons (2 saisons pluvieuses et 2 saisons sèches). La pluviométrie moyenne est de $1200 \mathrm{~mm}$ par an avec une température moyenne journalière qui varie de $25^{\circ} \mathrm{C}$ à $29^{\circ} \mathrm{C}$. On rencontre dans cette zone des sols ferralitiques, profonds et peu fertiles, des sols alluviaux et des vertisols (Dossa et al., 2015).

Le Département de l'Ouémé se caractérise par des sols ferralitiques, argileux-sableux, alluvionnaires et colluviaux, avec une végétation essentiellement anthropisée formée de fourrées, de quelques reliques forestières par endroits, une savane herbeuse, des prairies, des formations marécageuses à raphia et quelques mangroves (INSAE, 2002).

Le Département du Plateau est caractérisé par des sols ferrugineux tropicaux, les terres de barre sur le continental terminal profond et les sols très argileux profonds et humifères. Leclimat est de type soudano-guinéen. Les formations végétales sont la savane arborée/arbustive à dominance Daniela oliveri. On y retrouve aussi quelques reliques forestières par endroits (INSAE, 2002).

Le Département de l'Atlantique est pourvu d'un climat de type équatorial avec une alternance de deux saisons pluvieuses et deux saisons sèches. La pluviométrie moyenne est de $1200 \mathrm{~mm}$ par an, avec une température moyenne journalièrevariant de $25^{\circ} \mathrm{C}$ à $29^{\circ} \mathrm{C}$ et une humidité relative variant entre $69 \%$ et $97 \%$ (Sinsin et al. 2004).

Le Département du Littoral quant à lui se situe dans le secteur côtier. On y distingue des cordons littoraux récents en bordure de mer et des cordons littoraux anciens, entrecoupés par des lagunes et le complexe fluvio-lacustre de l'Ouémé, du lac Nokoué, du Couffo, du lac Ahémé et du Mono (Akoègninou et al. 2006). On y rencontre divers types de substrats de sables non ou très rarement inondés, sables périodiquement inondés, argiles à inondation prolongée, substrats submergés d'eau douce et substrats submergés d'eau saumâtres (Akoègninou et al. 2006).

\section{Méthode de collecte des données}

La méthodologie utilisée pour la collecte des données est celle d'une enquête rétrospective par entretien avec les acteurs de la filière élaborée après une première descente dans les marchés.L'enquête a été réalisée suivant une méthode d'interview structurée décrite par Dah-Nouvlessounonet al. (2015). Les données ont été collectées de mars 2017 à juillet 2017, directement auprès de 77 herboristes et 170 tradithérapeutes des Départements précités. Les données collectées étaient relatives aux caractéristiques socio-économiques des enquêtés, à la structure écologique de l'arbre de Annona muricata, aux différents organes utilisés et les maladies traitées, au mode d'emploi, etc. Le critère de choix et le nombre d'herboristes enquêtés dans un marché dépend 
de la richesse des étalages et de l'affluence du marché. Le critère de choix des tradithérapeutes réside dans la réputation et le savoir-faire de ces derniers.

\section{Méthode d'échantillonnage}

La taille de l'échantillon $(\mathrm{N})$ a été obtenue en utilisant l'approximation normale de la distribution binomiale proposée par Dagnelie (1998) :

$$
\mathrm{N}=\left[\left(\mathrm{U}_{1}-\alpha / 2\right)^{2} \times \mathrm{p}(1-\mathrm{p})\right] / \mathrm{d}^{2}
$$

$\mathrm{U}_{1}-\alpha / 2$ la valeur de la variable aléatoire normale pour la valeur de probabilité de $1-\alpha / 2, \alpha$ étant le risque d'erreur. Pour $\alpha=6 \%(1 \% \leq \mathrm{d} \leq 15 \%)$, la probabilité $1-\alpha / 2=0,975$ et on a $U_{1}-\alpha / 2=1,96$. P est la proportion d'acteurs (Herboristes et tradithérapeutes) qui utilisent la plante et d la marge d'erreur d'estimation, retenue à $6 \%$ dans cette étude.A partir des valeurs de $\mathrm{p}$ issues des résultats de la phase exploratoire de l'étude, au total 247 personnes ont été enquêtées. Ils ont été répartir en fonction de l'importance de l'utilisation de la plante. Dans chaque localité, les personnes enquêtées ont été identifiées selon un échantillonnage aléatoire simple.

\section{Méthode d'analyse statistique}

Les données collectées lors de l'enquête ont été codifiées, saisies et traitées avec le logiciel SPSS (Statistical Package for Social Sciences) version 20.0 (Norusis, 2002) pour la détermination des statistiques descriptives en termes de pourcentage et de moyenne. Les données quantitatives collectées au niveau des enquêtées ont été ensuite soumises à une analyse de la variance (ANOVA) en utilisant la procédure General Linear Model (PROC GLM)du logiciel SAS (StatisticalAnalysis System) version 9.2 selon Balogounet al. (2014). Les comparaisons de moyennesmultiples ont été réalisées avec le test deStudent Newman-Keuls (Dagnelie, 1986). Pour les classes d'âge, les intervalles suivants ont été considérés. Jeunes : 0 ans $\leq$ âge $\leq 35$ ans ; Adultes : 35 ans $<$ âge $\leq 60$ ans et Vieux : Age>60ans.

Pour chaque groupe d'enquêtés, le nombre de personnes ayant opté pour chacune des choix recensés a été calculé. Le tableau de contingence obtenu a été soumis à l'analyse factorielle des correspondances (AFC) simple à l'aide du logiciel Minitab 14 selon Belloet al. (2017). Les résultats des différentes analyses sont présentés sous forme de tableaux et de figures selon Kisauziet al. (2012).

\section{Résultats}

\section{Caractéristiques socio-démographiques des enquêtés}

Le tableau 1 présente le profil socio-économique des personnes enquêtées. Ilressort de l'analyse du tableau que les tradithérapeuteshommes (75\%) de l'ethnieGoun (27,1\%) et Cotafon(19,8\%) dominent les personnes 
enquêtées. Cestradithérapeutessont par ailleurs non scolarisés etâgés de plus de 60 ans. Ilspratiquent pour la majorité le christianisme.Le tableau 2 présente les résultats de l'analyse de variance et du test de Student-NewmanKeulsréaliséssur les donnéesquantitativesliées aux différentesethnies. L'analyse révèlequel'âge des personnesenquêtéesne varient pas significativement $(p>0,05)$ entre les ethnies. Cependant,les résultats d'enquête ont montré que les arbresplus âgés et plus gros sont retrouvés chez les Hôliet ceci de manière significative $(\mathrm{p}<0,05)$ comparée aux autresethnies (Tableau 2). En ce qui concerne les professions (tableau 3) l'analyse de variance et le test de Student-Newman-Keulsn'ontrévéléaucune différence significative $(p>0,05)$ entre les herboristes et les tradithérapeutes. Pour l'âge des arbres, et de leurs dimensions tenant compte des sites les abritant, l'analyserévèleque les tradithérapeutesvivent près des arbresplus grosque les herboristes $(\mathrm{p}<0,001)$ (tableau 3).

Tableau 1:Caractéristiques socio-économiques des enquêtées

\begin{tabular}{|c|c|c|}
\hline VARIABLES & MODALITES & FREQUENCE (\%) \\
\hline Profecsion & herboriste & 25,1 \\
\hline Proression & tradithérapeute & 74,9 \\
\hline \multirow{12}{*}{ Ethnie } & Adja & 9,7 \\
\hline & Aizô & 5,7 \\
\hline & Cotafon & 19,8 \\
\hline & Fon & 10,5 \\
\hline & Goun & 27,1 \\
\hline & Hôli & 0,8 \\
\hline & Mahi & 0,4 \\
\hline & Nago & 18,2 \\
\hline & Oueme & 1,2 \\
\hline & Peulh & 1,2 \\
\hline & Tori & 2,4 \\
\hline & Yoruba & 2,8 \\
\hline \multirow{3}{*}{ age (années) } & ]0;35] & 24,7 \\
\hline & ]35;60] & 35,2 \\
\hline & ]60;79] & 40,1 \\
\hline \multirow{4}{*}{ Niveau d'instruction } & non instruit & 40,1 \\
\hline & primaire & 23,4 \\
\hline & secondaire & 22,7 \\
\hline & supérieur & 13,8 \\
\hline \multirow{2}{*}{ Sexe } & femme & 25,1 \\
\hline & homme & 74,9 \\
\hline \multirow{3}{*}{ Religion } & animiste & 36,8 \\
\hline & chrétien & 55,9 \\
\hline & musulman & 7,3 \\
\hline
\end{tabular}


Tableau 2:Donnéesquantitativesliées aux ethnies

\begin{tabular}{ccccc}
\hline ETHNIE & Age(ans) & Taille-site(m) & $\begin{array}{c}\text { Estimation de l'âge des arbres } \\
\text { (années) }\end{array}$ & $\begin{array}{c}\text { Dimension des arbres } \\
\text { (cm) }\end{array}$ \\
\hline aizô & $59,64 \pm 3,55 \mathrm{a}$ & $6,86 \pm 0,5 \mathrm{ab}$ & $9,85 \pm 0,84 \mathrm{~b}$ & $43,64 \pm 2,51 \mathrm{~b}$ \\
oueme & $46,33 \pm 8,66 \mathrm{a}$ & $5,66 \pm 0,66 \mathrm{ab}$ & $10 \pm 2,51 \mathrm{~b}$ & $39 \pm 6,082 \mathrm{~b}$ \\
adja & $52,29 \pm 3,46 \mathrm{a}$ & $6,19 \pm 0,47 \mathrm{ab}$ & $9,85 \pm 0,98 \mathrm{~b}$ & $41,47 \pm 2,50 \mathrm{~b}$ \\
cotafon & $50,57 \pm 2,51 \mathrm{a}$ & $5,98 \pm 0,26 \mathrm{ab}$ & $9,35 \pm 0,67 \mathrm{~b}$ & $45,06 \pm 1,62 \mathrm{~b}$ \\
fon & $51,28 \pm 3,95 \mathrm{a}$ & $7,28 \pm 0,39 \mathrm{ab}$ & $9 \pm 0,56 \mathrm{~b}$ & $40,84 \pm 2,033 \mathrm{~b}$ \\
goun & $51,72 \pm 2,35 \mathrm{a}$ & $7 \pm 0,19 \mathrm{ab}$ & $10,16 \pm 0,50 \mathrm{~b}$ & $44,89 \pm 1,30 \mathrm{~b}$ \\
Hôli & $62,5 \pm 14,5 \mathrm{a}$ & $9,00 \pm 00 \mathrm{a}$ & $20,00 \pm 00 \mathrm{a}$ & $70,00 \pm 1,00 \mathrm{a}$ \\
mahi & $76 \pm 00 \mathrm{a}$ & $4,00 \pm 00 \mathrm{~b}$ & $6,00 \pm 00 \mathrm{~b}$ & $33,00 \pm 00 \mathrm{~b}$ \\
nago & $52,84 \pm 2,83 \mathrm{a}$ & $6,58 \pm 0,32 \mathrm{ab}$ & $8,53 \pm 0,34 \mathrm{~b}$ & $45,60 \pm 1,15 \mathrm{~b}$ \\
peulh & $43 \pm 4,35 \mathrm{a}$ & $6,33 \pm 0,88 \mathrm{ab}$ & $9,33 \pm 1,86 \mathrm{~b}$ & $48,33 \pm 10,92 \mathrm{~b}$ \\
tori & $47,83 \pm 7,01 \mathrm{a}$ & $7,16 \pm 0,87 \mathrm{ab}$ & $8,16 \pm 1,16 \mathrm{~b}$ & $49 \pm 6,16 \mathrm{~b}$ \\
yoruba & $61,42 \pm 6,15 \mathrm{a}$ & $8,28 \pm 0,35 \mathrm{a}$ & $10,85 \pm 0,96 \mathrm{~b}$ & $47 \pm 5,79 \mathrm{~b}$ \\
\hline f value & $1,09 \mathrm{~ns}$ & $2,12^{*}$ & $1,98^{*}$ & $1,94 *$ \\
\hline probabilité & 1,04 & 0,01 & 0,02 & 0,03
\end{tabular}

Les moyennes suivies des mêmes lettres alphabétiques ne sont pas significativement différentes $(p>0,05)$ d'après le test de Student Newman-Keuls.

Tableau3:Donnéesquantitativesliées aux professions

\begin{tabular}{ccccc}
\hline Profession & Age (Ans) & Taille-site & Estimation de l'âge des arbres & $\begin{array}{c}\text { Dimension des } \\
\text { arbres }\end{array}$ \\
\hline Tradipraticiens & $51,84 \pm 1,31 \mathrm{a}$ & $6,65 \pm 0,14 \mathrm{a}$ & $9,54 \pm 0,30 \mathrm{a}$ & $45,82 \pm 0,82 \mathrm{a}$ \\
Herboristes & $52,58 \pm 2,40 \mathrm{a}$ & $6,76 \pm 0,24 \mathrm{a}$ & $9,48 \pm 0,40 \mathrm{a}$ & $40,42 \pm 1,15 \mathrm{~b}$ \\
F Value & 0,08 & 0,13 & 0,01 & 11,92 \\
\hline probabilité & 0,79 & 0,72 & 0,91 & $\mathrm{p}<0,001$ \\
\hline
\end{tabular}

Les moyennes suivies des mêmes lettres alphabétiques ne sont pas significativement différentes $(P>0,05)$ d'après le test de Student Newman-Keuls.

\section{Différents organes utilisés dans des maladies par le traitement les catégories d'acteurs}

La figure 2 présente les différents organes utilisés pour le traitement des maladies. De l'analyse descriptive réalisée sur les différents organesde la plante utilisés pour le traitement des maladies (Figure 1), il ressort que les principaux organes d'Annona muricata utilisés pour le traitement des maladies sont majoritairement le fruits $(34 \%$,) le feuilles $(26 \%)$, l'écorces $(16 \%)$ et la racine $(24 \%)$. Ces différents organes sont vendus au marché en majorité par les herboristes $(54.7 \%)$. La période propice de vente est la période qui succède la fructification (56.3\%), et plus précisément dans les marchés $(73,3 \%)$. 


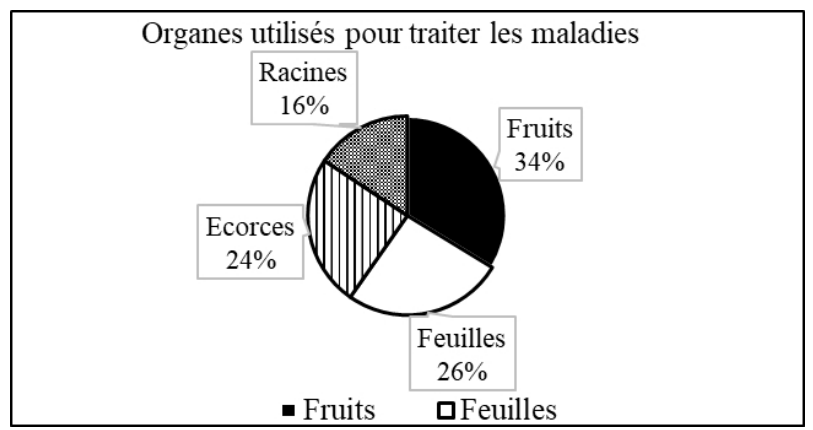

Fig. 2.Différents organes de la plante utilisés pour le traitement des maladies

Les résultats de l'Analyse Factorielle des Correspondances (AFC) réalisée sur les différents organes utilisés en lien avec les ethniesont permis de décrire les relations entre ces organes et les personnes enquêtées. Ces résultats indiquent que les deux premiers axes expliquent $100 \%$ de l'information totale.

La figure 3 présente le résultat de l'analyse factorielle des correspondances (AFC) réalisée sur les différents organes utilisés en lien avec es ethnies dans la zone d'étude. L'analyse de cette figure révèle que les personnes appartenant aux aires CulturellesOuémé utilisent les graines pour le traitement des maladies pendant que les Aïzo, les Fon, les Yorouba et les Mahi utilisent la tige pour traiter les maladies. Les autres ethnies notamment, les Tori, les Goun ainsi que les Adja et les Cotafonde même que les Yoruba, et Fon utilisent aussi bien les feuilles, les racines, les écorces ainsi que les fruits pour le traitement des maladies.Les Hôli et les Peulh, quant à eux utilisent particulièrement les feuilles pour le traitement des maladies. (Figure $3)$.

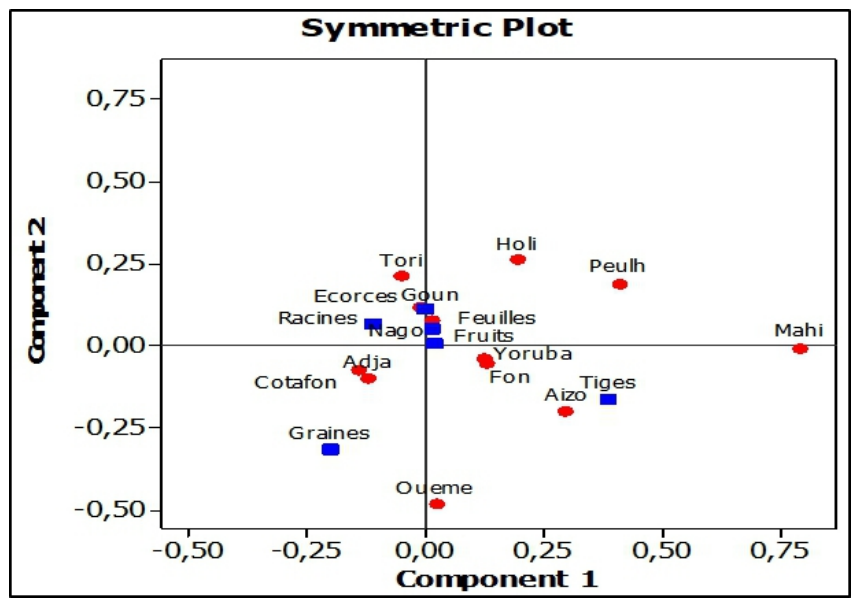

Fig. 3 : Organes utilisés en lien avec les ethnies: projection des organes et les groupes ethniques dans le système d'axes factoriels à l'issue d'une Analyse Factorielle des Correspondances (AFC). 
En ce qui concerne les catégories d'âge,les résultats de l'Analyse Factorielle des Correspondances (AFC) réalisée ont permis de décrire les relations entre ces organes et les classesd'âge. Ces résultats indiquent que les deux premiers axes expliquent $100 \%$ de l'information totale. La figure 4 présente le résultat de l'Analyse Factorielle des Correspondances (AFC) réalisée sur les différents organes utilisés en lien avec les catégories d'âge appartenant aux ethnies dans la zone d'étude. L'analyse de cette figure révèle que les personnes âgées utilisent les tiges et les graines de même que les fruits et les feuilles pour le traitement des maladies pendant que les adultes utilisent les écorces, les feuilles ainsi que les fruits ; par contre les jeunes utilisent plus les racines dans le traitement des maladies (figure 4).

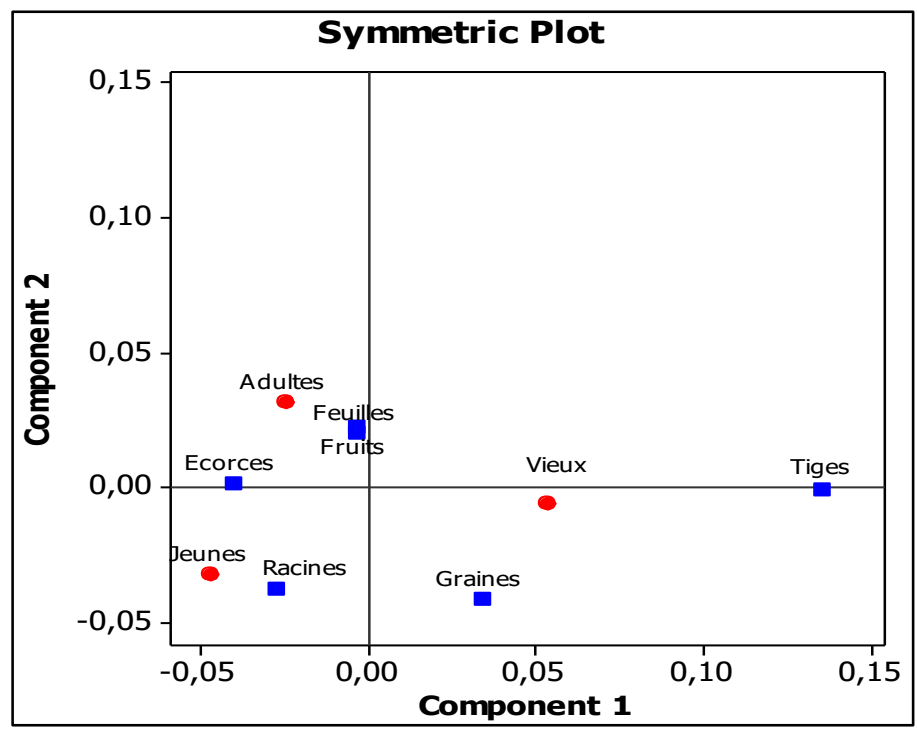

Fig. 4 : Organes utilisés en lien avec les catégories d'âge: projection des organes et les catégories d'âge dans le système d'axes factoriels à l'issue d'une Analyse Factorielle des Correspondances (AFC).

\section{Mode d'utilisation des organes}

La figure 5 présente le mode d'utilisation et de préparation des organes.Des résultats de l'analyse, les principaux modes de préparation des organes pour le traitement des maladies sont, la décoction, la tisane et la poudre. 


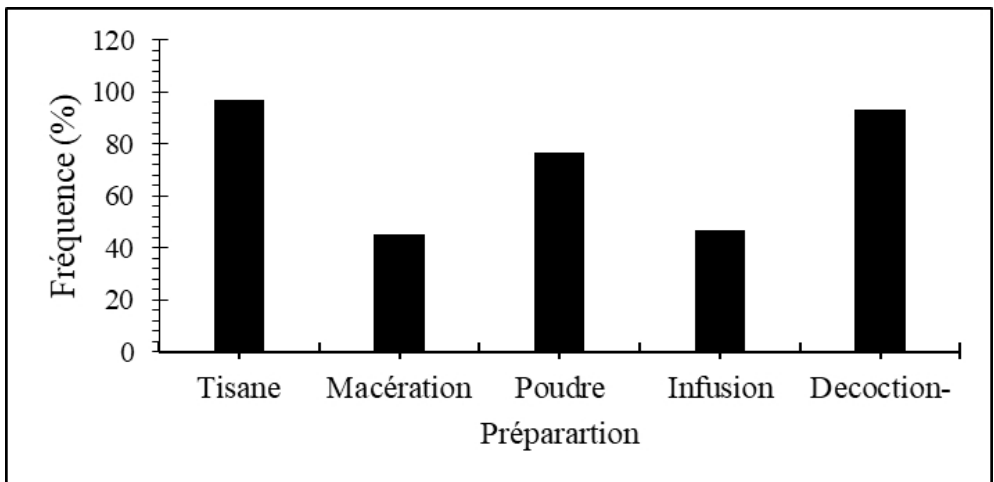

Fig 5 : Ordre d'appréciation et mode d'emploi des organes

Des modes d'utilisation selon les ethnies, les résultats de l'Analyse Factorielle des Correspondances (AFC) réalisée sur les différents modes d'utilisation des organes en lien avec les ethnies ont permis de décrire les relations entre ces organes et les classe d'âge. Ces résultats indiquent que les deux premiers axes expliquent $88,49 \%$ de l'information totale.

La figure 6 présente le résultat de l'analyse factorielle des correspondances (AFC) réalisée sur les différents modes d'utilisation des organes en lien avec les ethnies dans la zone d'étude. L'analyse de cette figure révèle que les personnes appartenant aux groupes ethniques Mahi, Ouémé, Nago et Goun font de la macération et de l'infusion pendant que les autres ethnies font de la décoction, la poudre et de la tisane pour le traitement des maladies (Figure 6).

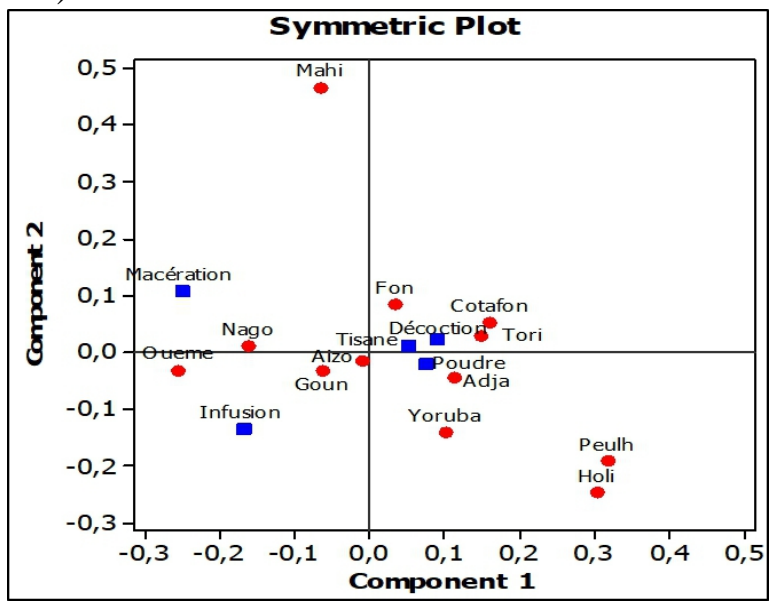

Fig 6 :Modes d'utilisation en lien avec les ethnies: projection des modes d'utilisation et les groupes ethniques dans le système d'axes factoriels à l'issue d'une Analyse Factorielle des Correspondances (AFC). 
Pour ce qui est des modes d'utilisation selon les classes d'âge, les résultats de l'Analyse Factorielle des Correspondances (AFC) réalisée sur les différents modes d'utilisation des organes en lien avec les classes d'âgeont permis de décrire les relations entre ces organes et les classe d'âge. Ces résultats indiquent que les deux premiers axes expliquent $100 \%$ de l'information totale. La figure 7 présente le résultat de l'analyse factorielle des correspondantes (AFC) réalisée sur les différents modes d'utilisation en lien avec les classes d'âges dans la zone d'étude.

L'analyse de cette figure révèleque les jeunes utilisent les organes de la plante sous forme d'infusion et de décoction. Les adultes utilisent les organes de la plante sous forme de tisane, de poudre et de décoction. Les personnes âgées, quant à elles en plus d'utiliser les organes des plantes sous les mêmes formes que les adultes, l'utilisent sous forme de macération.

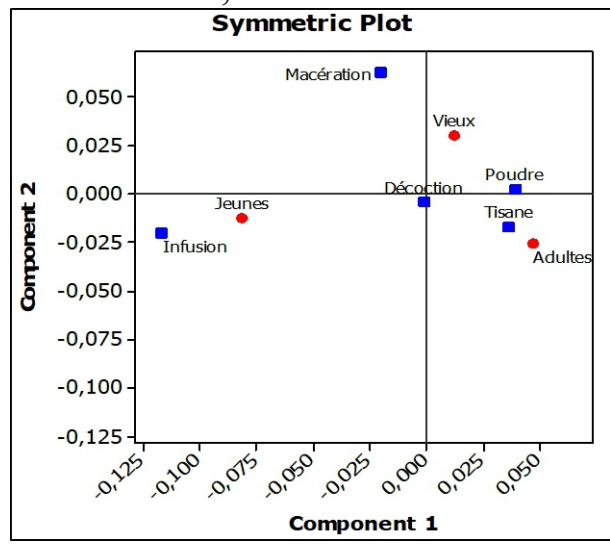

Fig. 7 : Modes d'utilisation en lien avec les classes d'âge: projection des modes d'utilisation et les classes d'âge dans le système d'axes factoriels à l'issue d'une Analyse Factorielle des Correspondances (AFC).

Les résultats de l'Analyse Factorielle des Correspondances (AFC) réalisée sur les différents modes d'utilisation des organes en lien avec les niveauxde scolarisationont permis de décrire les relations entre ces organes et les niveauxd'instruction. Ces résultats indiquent que les deux premiers axes expliquent $98,61 \%$ de l'information totale.La figure 8 présente les résultats de l'analyse factorielle des correspondances (AFC) réalisée sur les différents modes d'utilisation en lien avec le niveau d'instruction dans la zone d'étude. L'analyse de cette figure révèle que ceux qui ont le niveau supérieur utilisent les organes sous forme de poudre et de macération. Les personnes ayant le niveau primaire utilisent les organes de la plantesous forme de tisane alors que ceux ayant le niveau secondaire les utilisent sous forme de décoction. Les personnes non scolarisées utilisent les organes de la plante sous forme d'infusion 


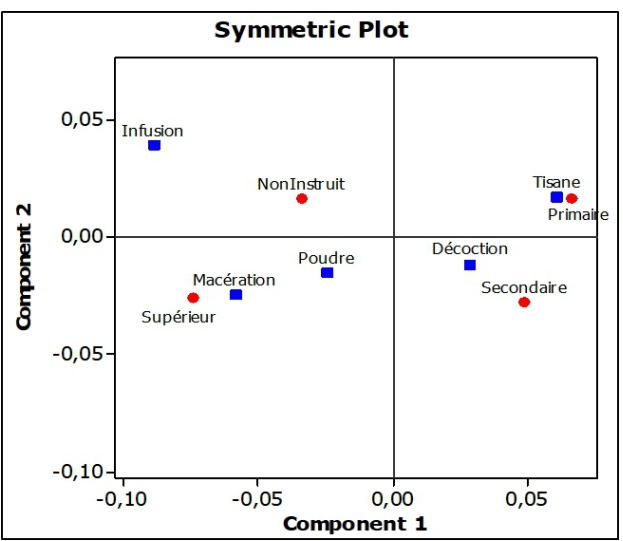

Fig 8 : Modes d'utilisation en lien avec les niveaux d'instruction: projection des modes d'utilisation et les niveaux d'instruction dans le système d'axes factoriels à l'issue d'une Analyse Factorielle des Correspondances (AFC)

\section{Maladiestraitées}

La figure 9 présente les différentes maladies traitées par les herboristes et les tradithérapeutes. L'analyse de cette figuremontre que la majorité des maladies traitées par la feuille de corossol sont l'insomnie, le cancer, le paludisme, la tension artérielle, la fièvre typhoïde $(51 \%)$.

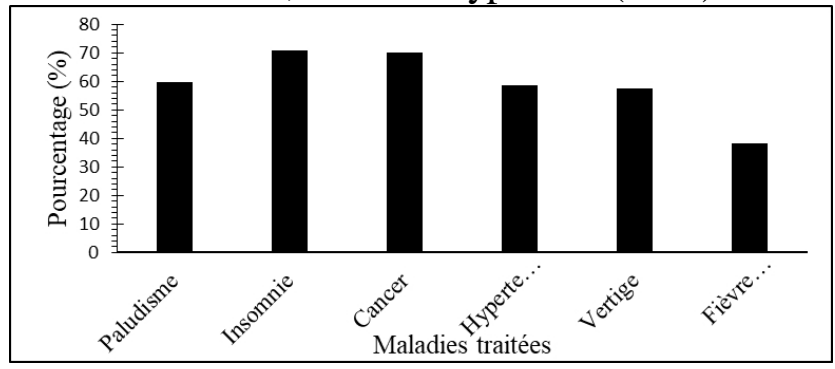

Fig. 9 : différentes maladies traitées

Les résultats de l'Analyse Factorielle des Correspondances (AFC) réalisée sur les différentes maladies en lien avec les ethnies ont permis de décrire les relations entre ces maladiestraitées et les ethnies. Ces résultats indiquent que les deux premiers axes expliquent $85,76 \%$ de l'information totale.La figure 10 présentele résultat de l'analyse factorielle des composantes (AFC) réalisé sur les différentes maladies traitées avec les organes du corossolier en lien avec les groupes ethniques. L'analyse de cette figure montre que les ethnies Holi, Tori, Mahi, Aizo Fon et Ouémé utilisent les feuilles de corossol pour traiter la tension artérielle et le vertige.Quant aux Peulh, ils les utilisent pour le traitement de la fièvre typhoïde. Les ethnies Goun, Yoruba,Cotafon et Nago par contre utilisent les organes pour traiter le paludisme, le cancer et l'insomnie (Figure 10). 


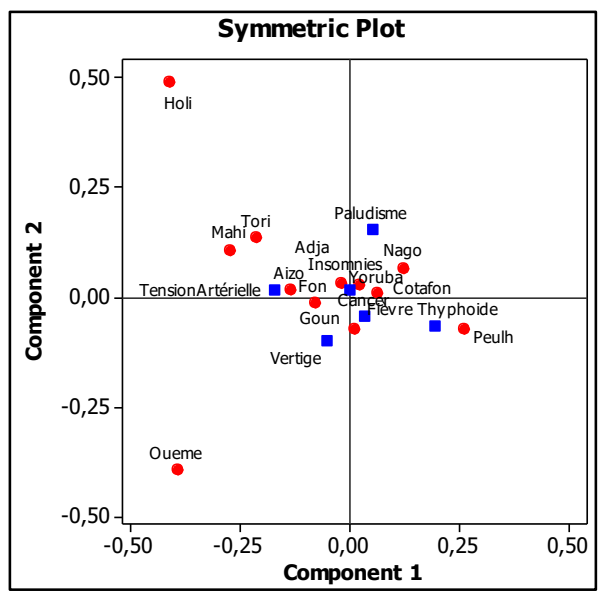

Fig.10 :Maladies traitées en lien avec les ethnies: projection des maladies traitées et les ethniesdans le système d'axes factoriels à l'issue d'une Analyse Factorielle des

Correspondances (AFC).

Les résultats de l'Analyse Factorielle des Correspondances (AFC) réalisée sur les différentes maladies en lien avec les niveauxd'instruction ont permis de décrire les relations entre les maladiestraitéeset les niveauxd'instruction. La figure 11 présente les résultats de l'analyse factorielle des composantes (AFC) réalisé sur les maladies traitées en lien avec le niveau d'instruction. Ces résultats indiquent que les deux premiers axes expliquent 94,34\% de l'information totale. Il ressort de l'analyse de cette figure que les non instruit utilisent les feuilles du corossolier pour traiter le cancer, la fièvre typhoïde et le vertige. Ceux qui ont le niveau du primaire utilisent les feuilles pour traiter la tension artérielle et l'insomnie. Ceux ayant le niveau secondaire utilisent la feuille pour soulager le paludisme et le cancer. 


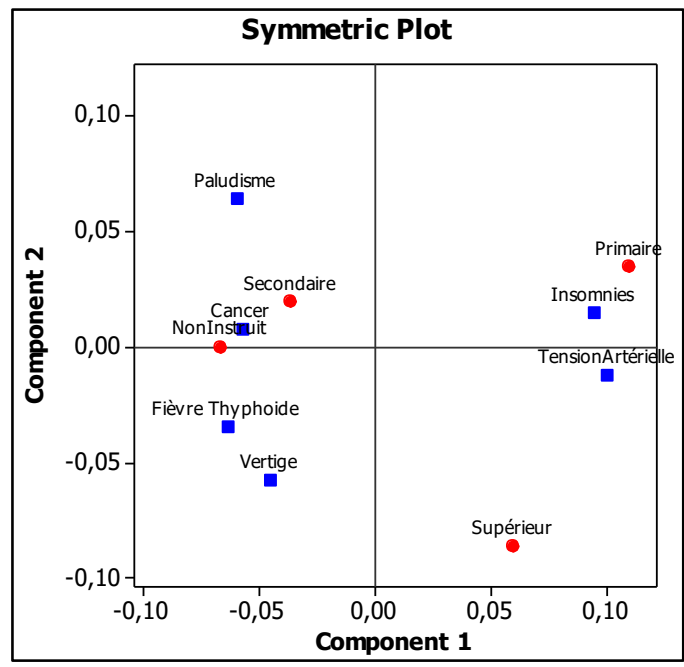

Fig. 11 : Maladies traitées en lien avec les niveaux d'instruction: projection des maladies traitées et les niveaux d'instructiondans le système d'axes factoriels à l'issue d'une Analyse Factorielle des Correspondances (AFC).

Les résultats de l'Analyse Factorielle des Correspondances (AFC) réalisée sur les différentes maladies en lien avec les classes d'âgeont permis de décrire les relations entre les maladiestraitéeset les classes d'âge. Ces résultats indiquent que les deux premiers axes expliquent $100 \%$ de l'information totale. La figure 12 présente les résultats de l'analyse factorielle des composantes (AFC) réalisées sur les différentes maladies traitées en lien avec les classes d'âge. Il ressort de cette analyse queles adultes utilisent la plante du corossol pour traiter l'insomnie, le cancer et la tension artérielle. Les jeunes l'utilisent beaucoup plus pour le traitement du paludisme et la fièvre typhoïde. Les personnes âgées utilisent la plante pour traiter le vertige. On retient de l'analyse de cette figure que les trois classes d'âge utilisent les feuilles du corossol dans le traitement de la fièvre de l'insomnie et du cancer. 


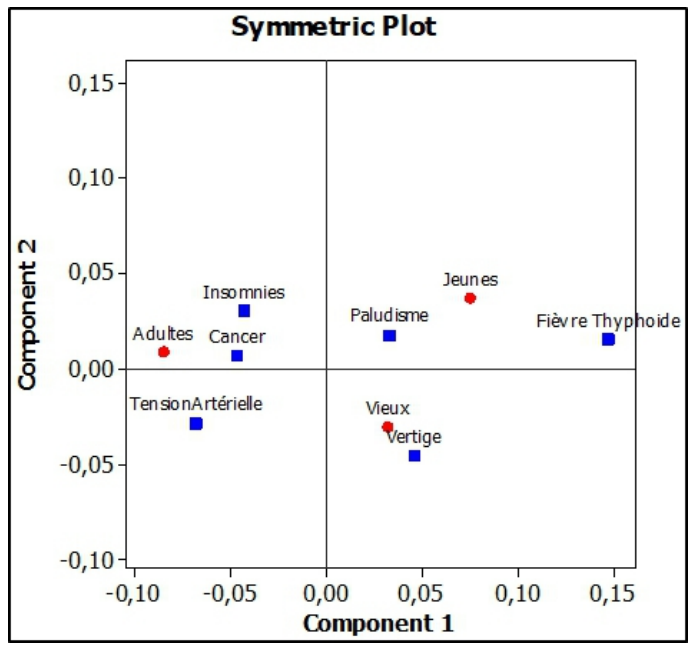

Fig. 12 :Maladies traitées en lien avec les classes d'âge: projection des Maladies traitées et les classes d'âgedans le système d'axes factoriels à l'issue d'une Analyse Factorielle des Correspondances (AFC).

\section{Discussion}

Cette étude a permis d'avoir une meilleure connaissance de l'utilisation des organes de la plante d'Annona muricata. Presque tous les organes de cette plante servent à différents usages. Le fruit en premier lieu intervient dans la nutrition de la population, avant d'être aussi utilisé pour la préparation des médicaments, c'est le premier facteur qui amène la population enquêtée à planter le corossolier dans les maisons. Les feuilles quant à elles sont comme les fruits dont la cueillette est plus accessible et ne porte pas trop atteinte à la plante elle-même. Par contre les organes de la plante (écorce, tige, racine) sont des parties très sensibles de la plante dont l'exploitation peut porter atteinte à la survie de la plante. Les graines quant à elles sont indispensables pour la germination et la reproduction de la plante, mais leur utilisation exagérée peut conduire la plante à la disparition

Les résultats de cette étude sont similaires à ceux de Sangare et al. (2012) qui démontrent que les feuilles sont sollicitées majoritairement au Maroc dans les traitements traditionnels; par contre ces résultats sont différents de ceux de Thirumalai et al.(2012) réalisés en Inde qui ont montré que la partie de la plante la plus utilisée est la tige feuillée. Cela peut s'expliquer par la différence entre les pathologies traitées et la zone d'étude. La feuille d' Annona muricata est utilisée dans le traitement de plusieurs maladies et plus particulièrement dans la traitement du cancer. Selon les informations tirées des résultats d'enquête, elle est utilisée sous forme de tisane (décoction).Cette méthode de préparation est similaire à celle obtenue par Gnagne etal., (2017).Elle l'est également à celle utilisée par les tradithérapeutes pour traiter les hépatites virales $\mathrm{B}$ et $\mathrm{C}$ dans les travaux 
réalisés par Guinninet al., (2015)toujours dans les départements du Sud Benin; ce qui montre une même identité culturelle traditionnelle que la population d'étude.Les résultats ont montré que la voie orale est la plus utilisée pour l'utilisation des produits issus de la préparation des décoctions ce qui est contraire aux résultats de Ouattara (2006) sur les plantes médicinales du bassin forestier de la Cote d'Ivoire qui montre que l'utilisation par voie orale des produits n'est que $32.25 \%$. Cette différence significative peut s'expliquer par les procédés de traitement des pathologies entre les groupes socioculturels enquêtés et plus précisément la différence entre les habitudes alimentaires de chaque région. Les travaux ont pris en compte 247 personnes composées de 170 tradithérapeutes et 77 herboristes d'une partie du sud Benin, la population de cette région est habituée à l'usage des organes des plantes pour le traitement des maladies ce qui confirme les données nationales qui stipulent que environ $80 \%$ de la population béninoise continuent de se soigner en recourant à la médecine traditionnelle. Les résultats sociodémographiques issus de cette enquête montrent que ce sont des personnes bien âgées, d'âge supérieur à 40 ans $\left(40,1 \%^{\circ}\right)$, qui pratiquent la médecine traditionnelle. Ces personnes fournissent des informations fiables, ce qui est relatif à leur expérience et ancienneté dans la pratique. Ils détiennent une partie importante du savoir ancestral qui est transmis oralement de génération en génération ; ce constat est similaire à ceux de Klotoé etal. (2013) et de Dassou et al. (2014) qui trouvent que les vertus médicinales des plantes sontdes connaissances ancestrales qui se transmettent de génération en génération. Ceci est d'autant plus justifiable que les herboristes, en majorité, ont signalé avoir hérité ces connaissances, comme le montraient les résultats de Dougnon et al.(2018)qui stipulent que les vendeuses de feuilles, particulièrement, sont des héritières du commerce des produits de la médecine traditionnelle. De même il ressort de ce travail que ce sont les personnes les plus démunies et de niveaux scolaires bas, qui fréquentent plus les tradithérapeutes pour leurs soins de santé. Cet aspect du travail confirme les données nationales sociodémographiques du Bénin, lesquelles données montrent clairement que ce sont les personnes démunies qui fuient les hôpitaux pour fautes de moyens financiers (Enquête démographique et de santé au Benin, 2017). Cet état de choses rejoint aussi les remarques faites par Lakouetené et al.(2009) qui déduisent dans le cas du paludisme à Bangui que la majorité des personnes atteintes ne se rendent pas à l'hôpital à cause de la cherté des médicaments. Mieux encore dans cette étude-ci, il s'agit d'une maladie comme le cancer dont le traitement dans les hôpitaux est plus coûteux que le traitement du paludisme.

Les résultats montrent que la majorité des herboristes et des tradithérapeutes sont des personnes non scolarisées $(40,1 \%)$.Ces résultats sont similaires aux données nationales qui montrent selon le quatrième rapport national du Bénin sur la diversité biologique, que les populations rurales 
majoritairement analphabètes sont détentrices des connaissances médicales des plantes (PNUD-Bénin, 2009).Aussi, les résultats concernant les religions montrent que les herboristes et les tradithérapeutes sont constitués en majorité des chrétiens (55.8\%); ce qui montre que l'usage de la médecine traditionnelle n'a rien de diabolique. Selon les travaux réalisés par Zinsou et al., (1990) qui indiquent que le faible taux de présence des malades de cancer est $\mathrm{du}$ fait que les malades et leurs parents pensent qu'il existe une transgression des ancêtres s'adressant souvent aux tradithérapeutes. Les résultats ont montré que les herboristes sont uniquement des femmes âgées d'au moins 30 ans et ayant une expérience professionnelle d'au moins 10 ans Cela s'explique par le fait qu'en Afrique et particulièrement au Bénin, les activités de vente d'articles au marché sont habituellement réservées aux femmes. Ces résultats sont similaires à ceux de Adomou et al,(2011) qui montrent que l'étalage de vente des feuilles dans les marchés est souvent la propriété des femmes.

De l'utilisation abusive des organes de plantes et de la rareté d'autres espèces, l'idée de conservation et de protection des plantes qui sont utilisées dans le traitement des maladies commence par apparaître dans le comportement de la population afin de sauvegarder le patrimoine hérité des parents. Ce comportement est aussi démontré par les travaux de Klotoé ét al, (2013)où les femmes herboristes sont en partenariat avec des fermiers qui reproduisent les plantes qu'ils mettent en combinaison pour le traitement du diabète. Cette étude retrace une nécessité de faire des recherches phytochmique, toxicologique et pharmacologique sur la feuille d'Annona muricata afin de la rendre utile à la communauté.

\section{Conclusion}

Cette enquête ethnobotanique a permis de savoir qu'au-delà de la littérature, les organes de la plante Annona muricata sont utilisés dans le traitement des maladies au sud Benin. Principalement la feuille qui intervient dans le traitement thérapeutique du cancer. Des expériences (activité anti oxydante anti inflammatoire et analgésique) seront réalisées au laboratoire sur les extraits de la feuille d'Annona muricata afin de donner une valeur plus scientifique à ce travail. Le screening phytochimique de même que la recherche des métabolites secondaires contenus dans les extraits semi éthanolique et éthanolique sera réalisé Des résultats issus de ce travail, la population sera sensibilisée pour mettre l'usage des organes de la plante d'Annona muricata dans leur comportement alimentaire dans le seul souci de prévenir les pathologies. 


\section{References :}

1. Adjanohoun J.E., Aboubacar N., Dramane K., Ebot M.E., Ekpere J.A., Enow-Orock E.G., Focho D., Gbile Z.O., Kamanyi A., Kamsu K.J., Keita A., Mbenku T., Mbi C.N., Mbiele A.L., Mbome I.L., Mubiru N.K., Nancy W.L., Kongmeneck,B., Satabie B., Sofora A., Tamze V., \&Wirmun C.K. (1998). Traditional medicine and pharmacopoeia.Contribution to ethnobotanical and floristic studies in Cameroon.Organisation of Africanunityscientific and research commission. Centre national de production de manuels scolaire. PortoNovo p. 227.

2. Adomou, A.C., Agbani O.P., Sinsin B. ,2011. Plantes In Neuenschwander P., Sinsin B., \&Goergen G. (eds) Protection de la Nature en Afrique de l'Ouest : Une Liste Rouge pour le Bénin. Nature Conservation in West Africa: Red List for Benin, International Institute of Tropical Agriculture, Ibadan (Nigeria), 21-46.

3. 3.Agbankpé, A.J., Dougnon T.V., Bankolé H.S., Houngbegnon O., Dahnouvlessounon D., \&Baba-Moussa L., 2016.In vitro Antibacterial Effects of Cratevaadansonii, Vernoniaamygdalina and Sesamumradiatum Used for the Treatment of Infectious Diarrhoeas in Benin, Journal of Infectious Diseases \& Therapy, 4: 281.

4. 4.Agbankpé, A.J., Dougnon T.V., Bankolé H.S., Yèhouénou B., Yédomonhan H., Lègonou M., \&Dougnon T.J., 2014.Etude ethnobotanique des légumes feuilles thérapeutiques utilisés dans le traitement des diarrhées au sud-Bénin (Afrique de l'Ouest), International Journal of Biological and Chemical Sciences, 8(4): 17841795 .

5. .Akoegninou, A., 2012. Étude ethnobotanique des plantes médicinales vendues dans le marché d'Abomey-Calavi au Bénin. International Journal of Biological and Chemical Sciences, 6(2): 745-772. DOI

6. 6 Akoègninou, A., Van der Burg W.J., \&Van der Maesen L.J.G., 2006.Flore analytique du Bénin. BackhuysPublishers, 1043p

7. Aliam, UICC, 2017. Aliance des Ligues Francophone et Méditerraneen L'union Internationale Contre Le Cancer : Cancer and Non Communicable Diseases in Africa

8. Balogoun, I., Saidou A., Ahoton E.L., Amadji G.L., Ahohuendo C.B., Adebo J.B., Babatoundé S., Chougourou D., Adoukonou S.H.,\& Ahanchédé A., 2014. Caractérisation des systèmes de production à base d'anacardier dans les principales zones de culture au Bénin. Agronomie africaine.26 (1) : - 9-22.

9. Bello,O.D., Ahoton, L.E., Saidou, A. Akponikpè,I.P.B.Ezin,V.A., Balogoun, I., Aho, N., 2017. Climate change and cashew (AnacardiumOccidentale L.) productivity in Benin: perceptions, 
endogenous measures of adaptation. Int. J. Biol. Chem. Sci. 11(3): 924-946.

10. Dagnelie P., 1986. Théorie et méthodes statistiques. Applications agronomiques. Vol 2. Les presses agronomiques de Gembloux. A.S.B.L. (Belgique). 463 p.

11. Dagnelie, P., 1998. Statistique théorique et appliquée. Tome 2 : Inférences statistiques à une et deux dimensions. De Boeck et Larcier. Paris-Bruxelles. France Belgique. 659 p.

12. Dah-Nouvlessounon Durand, Adoukonou-Sagbadja Hubert, DiarrassoubaNafan, AdjanohounAdolphe, Baba-Moussa Farid, Sezan Alphonse,\& Baba-Moussa L., 2015:Indigenous knowledge and socioeconomic values of three kola species (Cola nitida, Colaacuminata and Garcinia kola) used in southern Benin. European Scientific Journal.11 (36): 206-227.

13. Dassou H.G., Ogni C.A., Yédomonhan H., Adomou A.C., Tossou M., Dougnon J.T., \&Akoègninou A., 2014.Diversité, usages vétérinaires et vulnérabilité des plantes médicinales au Nord-Bénin, International Journal of Biological and Chemical Sciences, 8(1): 189-210.

14. Dossa k. Toni H., Azonanhoun P. and \& Djossa A.B., 2015. Caractérisation de quelques peuplements naturels de Baobab (Adansoniadigitata L.) et des pressions subies dans les différentes zones chorologiques du Bénin. Journal of AppliedBioscience. 93: 8760-8769.

15. Dougnon, G., 2013. Etudes ethnobotanique et ethno-pharmacologique des plantes utilisées dans le traitement traditionnel de l'hypertension artérielle au centre-Bénin, Thèse de Doctorat d'Etat en Pharmacie, Université d'AbomeyCalavi, Faculté des Sciences de la Santé, 202 p.

16. Guinnin F. D. Félix, T. Isabelle SACRAMENTO, Alphonse SEZAN et Jean-Marc ATEGBO. Etude Ethnobotanique des plantes médicinales utilisées dans le traitement traditionnel des hépatites virales $\mathrm{B}$ et $\mathrm{C}$ dans quelques départements du Bénin

17. Globocan, 2012. Global cancer statistics.Breast Cancer Estimated Incidence, Mortality and Prevalence Worldwide in 2012

18. Gnagne, Y.A., Yapo B. O., Mambo V., Meite L.,\& Houenou P. 2013. Pollution urbaine et processus d'acidification des eaux de puits de la ville d'Abidjan, Côte d'Ivoire. J. Soc. Ouest-Afr. Chim. 03:55 - 61

19. INSAE, 2004. RGPH2 Cahier des villages et quartiers de ville Département de l'OUEME, Bénin 22p

20. Kisauzi T., Mangheni M.N., Seguya H., \&Bashaasha B., 2012. Gender dimensions of farmer's perceptions and knowledge on climate change in Teso sub-region. Eastern Uganda. AfricanCrop Science Journal. 20(2): $275-286$. 
21. Klotoé J.R., 2016. Plantes hémostatiques du sud-Bénin : Etude ethnopharmacologique et propriétés hémostatiques, Thèse de doctorat unique, Université d'Abomey-Calavi, Bénin, p. 132.

22. Klotoé J.R., Dougnon T.V., Koudouvo K., Atègbo J.M., Loko .F, Akoègninou A., Aklikokou K., Dramane K., \& Gbeassor M., 2013.Ethnopharmacological survey on antihemorrhagicmedicinal plants in South of Benin. European Journal of Medicinal Plants, 3(1): 40-51

23. Lakouéténé, D.P.B., Ndolngar G., Berké B., Moyen J.M., Komba E.K., Zinga I., Silla S.,Millogo-Rasolodimby J., Vincendeau P., SyssaMagalé J.L., Nacoulma-Ouedrago O.G.,Laganie R., Badoc A., \&Chèze C., 2009.Enquête ethnobotanique des plantes utilisées dans le traitement du paludisme à Bangui, Bull. Soc. Pharm., 148, 12313824.Norusis, M.J., 2002.SPSS 11.0 guide to data analysis. Prentice and Hall.

24. Thirumalai T, Beverly CD, Sathiyaraj K,Senthilkumar B, David E. 2012Ethnobotanical Study of anti-diabetic medicinal plants used by the localpeople in javadhu hills Tamilnadu, India. AsianPacific Journal of Tropical Biomedicine,2(2): S910-S913.

25. 25 OMS, 2014. Statistiques sanitaires mondiales 2014 de l'OMS. www.who.int/fr.

26. OMS, 2018. Fièvre typhoïde. http://www.who.int/features/qa/typhoidfever/fr/ (consulté le 14 juillet 2018)

27. ONU, 2011. Mécanisme de suivi des engagements pris en faveur du développement de l'Afrique

28. Ouattara, D. 2006. Contribution à l'inventairedes plantes médicinales significatives utilisées dans la région de Divo (Sud forestier de la Côte d'Ivoire) et à la diagnose du poivrier de Guinée : Xilopia

29. 29.PNUD Bénin, 2009. Quatrième rapport national du Bénin sur la diversité biologique, Ministère de l'Environnement et de la Protection de la Nature, PNUD Bénin, 172 p.

30. Sangare M. M, Sina H, Dougnon J, BaléB,Atègbo J-M, Dramane K. 2012. Etudeethnobotanique des plantes hépatotropes et de l'usage traditionnel de GomphrenacelosioidesMart. (Amaranthaceae) au Bénin. Int J. Biol. Chem Sci ., 6(6): 5008-5021.

31. Sinsin, B., Eyog-Matig O., Sinadouwirou Th., \&Assogbadjo A., 2004.Dendrometric characteristics as indicators of pressure of Afzelia Africana Sm. Dynamic changes in treesfound in differentclimate zones of Benin. Biodiversity and Conservation, 13(8): 1555-1570

32. World Health Organization, 2004.Web page http://www.who.org (consultéenjanvier 2018). 